PROCEEDINGS OF THE

AMERICAN MATHEMATICAL SOCIETY

Volume 135, Number 7, July 2007, Pages 1993-2002

S 0002-9939(07)08793-X

Article electronically published on March 2, 2007

\title{
HILBERT FUNCTIONS OF GORENSTEIN MONOMIAL CURVES
}

\author{
FEZA ARSLAN AND PINAR METE
}

(Communicated by Bernd Ulrich)

\begin{abstract}
It is a conjecture due to M. E. Rossi that the Hilbert function of a one-dimensional Gorenstein local ring is non-decreasing. In this article, we show that the Hilbert function is non-decreasing for local Gorenstein rings with embedding dimension four associated to monomial curves, under some arithmetic assumptions on the generators of their defining ideals in the noncomplete intersection case. In order to obtain this result, we determine the generators of their tangent cones explicitly by using standard basis computations under these arithmetic assumptions and show that the tangent cones are Cohen-Macaulay. In the complete intersection case, by characterizing certain families of complete intersection numerical semigroups, we give an inductive method to obtain large families of complete intersection local rings with arbitrary embedding dimension having non-decreasing Hilbert functions.
\end{abstract}

\section{INTRODUCTION}

In this article, we study the Hilbert function of a one-dimensional Gorenstein local ring of embedding dimension four associated to a Gorenstein monomial curve. The Hilbert function of a local ring $R$ with maximal ideal $m$ is the numerical function defined as $H_{R}(n)=\operatorname{dim}_{R / m}\left(m^{n} / m^{n+1}\right)$ for all $n \geq 0$, while the embedding dimension of $R$ is $H_{R}(1)$. In other words, the Hilbert function of a local ring $R$ with maximal ideal $m$ is defined as the Hilbert function of its associated graded ring with respect to $m$ (which is $\operatorname{gr}_{m}(R)=\bigoplus_{i=0}^{\infty} m^{i} / m^{i+1}$ ). In general, very little is known about the behavior of the Hilbert function of a Cohen-Macaulay local ring, since its associated graded ring can be very bad [19. The main open problem in the one-dimensional case was Sally's conjecture [18]:

Sally's conjecture. If $R$ is a one-dimensional Cohen-Macaulay local ring with small enough embedding dimension, then $H_{R}(n)$ is nondecreasing.

The conjecture is obvious for embedding dimension one, in which case $R$ is regular and $H_{R}(n)=1$ for all $n$. Matlis proved Sally's conjecture for embedding dimension two by showing that $H_{R}(n)=\min \{n+1, e(R)\}$, where $e(R)$ is the multiplicity of the local ring $R$ 14. Finally, Elias proved the conjecture for the embedding dimension three case [5]. In the literature, there are several examples

Received by the editors December 17, 2005 and, in revised form, April 1, 2006.

2000 Mathematics Subject Classification. Primary 13H10, 14H20; Secondary 13P10.

Key words and phrases. Gorenstein local ring, Hilbert function of a local ring, tangent cone, monomial curve, numerical semigroup, standard basis.

The second author was supported by TÜBİTAK with grant no. TBAG-HD/108(105T543). 
showing that Cohen-Macaulayness of a one-dimensional local ring does not necessarily assure the non-decreasing behavior of its Hilbert function for embedding dimensions greater than three. The first examples of local rings with decreasing Hilbert function were given by Herzog-Waldi [12] and Eakin-Sathaye [7. Furthermore, Orecchia showed that for all $b \geq 5$ there exists a reduced one-dimensional local ring of embedding dimension $b$ with decreasing Hilbert function [16]. In the remaining case of embedding dimension four, similar examples were given by Gupta and Roberts in [10. Thus, the Cohen-Macaulayness of a one-dimensional local ring with embedding dimension greater than three does not guarantee that its Hilbert function is non-decreasing. However, it is a conjecture due to M. E. Rossi, that a one-dimensional Gorenstein local ring (a Cohen-Macaulay ring of type 1) has a non-decreasing Hilbert function, and this problem is open even for Gorenstein local rings with embedding dimension four associated to Gorenstein monomial curves in affine 4-space [6]. These curves are our main geometric objects of interest in this article.

We recall that a monomial affine curve $C$ has a parametrization

$$
x_{1}=t^{n_{1}}, x_{2}=t^{n_{2}}, \ldots, x_{d}=t^{n_{d}}
$$

where $n_{1}, n_{2}, \ldots, n_{d}$ are positive integers with $\operatorname{gcd}\left(n_{1}, n_{2}, \ldots, n_{d}\right)=1$. A numerical semigroup denoted by $\left\langle n_{1}, n_{2}, \ldots, n_{d}\right\rangle$ is given as $\left\langle n_{1}, n_{2}, \ldots, n_{d}\right\rangle=\{n \mid n=$ $\sum_{i=1}^{d} a_{i} n_{i}, a_{i}$ 's are non-negative integers $\}$ where $\operatorname{gcd}\left(n_{1}, n_{2}, \ldots, n_{d}\right)=1$. A numerical semigroup $\left\langle n_{1}, n_{2}, \ldots, n_{d}\right\rangle$ is symmetric if and only if the number of gaps is equal to the number of nongaps $\left(n \notin\left\langle n_{1}, n_{2}, \ldots, n_{d}\right\rangle\right.$ with $0<n \leq c$ is called a gap, while $n \in\left\langle n_{1}, n_{2}, \ldots, n_{d}\right\rangle$ with $0 \leq n<c$ is called a nongap, where $c$ is the greatest integer not in the semigroup). The algebraic characterization of symmetric semigroups given by Kunz implies that $\left\langle n_{1}, n_{2}, \ldots, n_{d}\right\rangle$ is symmetric if and only if $k\left[\left[t^{n_{1}}, t^{n_{2}}, \ldots, t^{n_{d}}\right]\right]$ is Gorenstein, where $k$ is the underlying field of the affine space, see 13. A monomial curve given by the parametrization in (1.1) is called a Gorenstein monomial curve if the associated local ring $k\left[\left[t^{n_{1}}, t^{n_{2}}, \ldots, t^{n_{d}}\right]\right]$ is Gorenstein, or equivalently, if $\left\langle n_{1}, n_{2}, \ldots, n_{d}\right\rangle$ is symmetric.

Let $C$ be a monomial curve having the parametrization

$$
x_{1}=t^{n_{1}}, x_{2}=t^{n_{2}}, x_{3}=t^{n_{3}}, x_{4}=t^{n_{4}}
$$

for which the symmetric numerical semigroup $\left\langle n_{1}, n_{2}, n_{3}, n_{4}\right\rangle$ is minimally generated by $n_{1}, n_{2}, n_{3}, n_{4}$. (Clearly, this assures that the Gorenstein local ring $k\left[\left[t^{n_{1}}, t^{n_{2}}, t^{n_{3}}, t^{n_{4}}\right]\right]$ has embedding dimension four.) Thus, in this case, we also say that $C$ is a Gorenstein monomial curve with embedding dimension four. In 1975 , Bresinsky not only showed that the ideal $I(C)$ of the curve $C$ is minimally generated by either 3 (complete intersection case) or 5 (non-complete intersection case) elements, but also gave an explicit description of the defining ideal $I(C)$, see [3]. Knowing the defining ideal $I(C)$ explicitly by the work of Bresinsky, we investigate the generators of the ideal $I(C)_{*}$, which is generated by the polynomials $f_{*}$ for $f$ in $I(C)$, where $f_{*}$ is the homogeneous summand of $f$ of least degree. $I(C)_{*}$, which is the defining ideal of the tangent cone of $C$ at 0 can be used to find the Hilbert function of $k\left[\left[t^{n_{1}}, t^{n_{2}}, t^{n_{3}}, t^{n_{4}}\right]\right]$, since $g r_{m}\left(k\left[\left[t^{n_{1}}, t^{n_{2}}, t^{n_{3}}, t^{n_{4}}\right]\right]\right)$ is isomorphic to the ring $k\left[x_{1}, x_{2}, x_{3}, x_{4}\right] / I(C)_{*}$. Based on the standard basis theory, we find the generators of the tangent cone of a Gorenstein monomial curve $C$ with embedding dimension four, under some arithmetic conditions on the generators of its defining ideal. Showing the Cohen-Macaulayness of the tangent cones of these families of 
Gorenstein monomial curves gives us the opportunity to obtain families of Gorenstein local rings with non-decreasing Hilbert function in the embedding dimension four case.

\section{NON-COMPLETE INTERSECTION CASE}

In this section, we first recall Bresinsky's theorem, which gives the explicit description of the defining ideal of a Gorenstein monomial curve with embedding dimension four in the non-complete intersection case [3, Theorem 3].

Theorem 2.1 (Bresinsky's Theorem). Let $C$ be a monomial curve having the parametrization

$$
x_{1}=t^{n_{1}}, x_{2}=t^{n_{2}}, x_{3}=t^{n_{3}}, x_{4}=t^{n_{4}}
$$

where $S=\left\langle n_{1}, n_{2}, n_{3}, n_{4}\right\rangle$ is a numerical semigroup minimally generated by $n_{1}, n_{2}, n_{3}, n_{4}$. The semigroup $\left\langle n_{1}, n_{2}, n_{3}, n_{4}\right\rangle$ is symmetric and $C$ is a non-complete intersection curve if and only if $I(C)$ is generated by the set

$$
\begin{aligned}
G=\left\{f_{1}=\right. & x_{1}^{\alpha_{1}}-x_{3}^{\alpha_{13}} x_{4}^{\alpha_{14}}, f_{2}=x_{2}^{\alpha_{2}}-x_{1}^{\alpha_{21}} x_{4}^{\alpha_{24}}, f_{3}=x_{3}^{\alpha_{3}}-x_{1}^{\alpha_{31}} x_{2}^{\alpha_{32}}, \\
& \left.f_{4}=x_{4}^{\alpha_{4}}-x_{2}^{\alpha_{42}} x_{3}^{\alpha_{43}}, f_{5}=x_{3}^{\alpha_{43}} x_{1}^{\alpha_{21}}-x_{2}^{\alpha_{32}} x_{4}^{\alpha_{14}}\right\}
\end{aligned}
$$

where the polynomials $f_{i}$ are unique up to isomorphism and $0<\alpha_{i j}<\alpha_{j}$. Here $\alpha_{i}$ is the smallest integer such that $\alpha_{i} n_{i} \in\left\langle n_{1}, \ldots, \hat{n}_{i}, \ldots, n_{4}\right\rangle$, where $\hat{n}_{i}$ denotes that $n_{i} \notin\left\langle n_{1}, \ldots, \hat{n}_{i}, \ldots, n_{4}\right\rangle$.

Remark 2.2. Moreover, in the same article, Bresinsky also shows that the semigroup $S=\left\langle n_{1}, n_{2}, n_{3}, n_{4}\right\rangle$ is symmetric and the defining ideal is as in Theorem 2.1 if and only if $n_{1}=\alpha_{2} \alpha_{3} \alpha_{14}+\alpha_{32} \alpha_{13} \alpha_{24}, n_{2}=\alpha_{3} \alpha_{4} \alpha_{21}+\alpha_{31} \alpha_{43} \alpha_{24}, n_{3}=\alpha_{1} \alpha_{4} \alpha_{32}+$ $\alpha_{14} \alpha_{42} \alpha_{31}, n_{4}=\alpha_{1} \alpha_{2} \alpha_{43}+\alpha_{42} \alpha_{21} \alpha_{13}$ with $\operatorname{gcd}\left(n_{1}, n_{2}, n_{3}, n_{4}\right)=1, \alpha_{i}>1,0<$ $\alpha_{i j}<\alpha_{j}$ for $1 \leq i \leq 4$ and $\alpha_{1}=\alpha_{21}+\alpha_{31}, \alpha_{2}=\alpha_{32}+\alpha_{42}, \alpha_{3}=\alpha_{13}+\alpha_{43}$, $\alpha_{4}=\alpha_{14}+\alpha_{24}[3$, Theorem 5].

Remark 2.3. Theorem 2.1 implies that for any non-complete intersection Gorenstein monomial curve with embedding dimension four, the variables can be renamed to obtain generators exactly of the given form, and this means that there are six isomorphic possible permutations which can be considered within three cases:

(1) $f_{1}=(1,(3,4))$

(a) $f_{2}=(2,(1,4)), f_{3}=(3,(1,2)), f_{4}=(4,(2,3)), f_{5}=((1,3),(2,4))$

(b) $f_{2}=(2,(1,3)), f_{3}=(3,(2,4)), f_{4}=(4,(1,2)), f_{5}=((1,4),(2,3))$

(2) $f_{1}=(1,(2,3))$

(a) $f_{2}=(2,(3,4)), f_{3}=(3,(1,4)), f_{4}=(4,(1,2)), f_{5}=((2,4),(1,3))$

(b) $f_{2}=(2,(1,4)), f_{3}=(3,(2,4)), f_{4}=(4,(1,3)), f_{5}=((1,2),(4,3))$

(3) $f_{1}=(1,(2,4))$

(a) $f_{2}=(2,(1,3)), f_{3}=(3,(1,4)), f_{4}=(4,(2,3)), f_{5}=((1,2),(3,4))$

(b) $f_{2}=(2,(3,4)), f_{3}=(3,(1,2)), f_{4}=(4,(1,3)), f_{5}=((2,3),(1,4))$

Here, the notation $f_{i}=(i,(j, k))$ and $f_{5}=((i, j),(k, l))$ denote the generators $f_{i}=x_{i}^{\alpha_{i}}-x_{j}^{\alpha_{i j}} x_{k}^{\alpha_{i k}}$ and $f_{5}=x_{i}^{\alpha_{k i}} x_{j}^{\alpha_{l j}}-x_{k}^{\alpha_{j k}} x_{l}^{\alpha_{i l}}$. Thus, given a Gorenstein monomial curve having parametrization as in (1.2), if we have the extra condition $n_{1}<n_{2}<n_{3}<n_{4}$, then the generator set of its defining ideal is exactly given by one of these six permutations. 
From our computations with Macaulay [2] and Singular [9, we have observed that there are many non-complete intersection Gorenstein monomial curves with embedding dimension four having Cohen-Macaulay tangent cones generated by exactly five elements, and moreover, the generator set of each of these curves turned out to be a standard basis with respect to at least one local degree ordering. Thus, by determining the common arithmetic conditions satisfied by the generators of the defining ideals of these monomial curves, we apply the standard basis algorithm in 8 to the set of generators given by Bresinsky satisfying these arithmetic conditions and show that there is a large family of non-complete intersection Gorenstein monomial curves having Cohen-Macaulay tangent cones, which guarantees a non-decreasing Hilbert function.

Proposition 2.4. Let $C$ be a non-complete intersection Gorenstein monomial curve having parametrization as in (1.2) with $n_{1}<n_{2}<n_{3}<n_{4}$, and let the generators of $I(C)$ be given by the set $G$ in Theorem 2.1. If $\alpha_{2} \leq \alpha_{21}+\alpha_{24}$, then the defining ideal $I(C)_{*}$ of the tangent cone is generated by a set $G_{*}$ consisting of the least homogeneous summands of the $f_{i}$ 's in $G$ for $1 \leq i \leq 5$.

Remark 2.5. The condition $n_{1}<n_{2}<n_{3}<n_{4}$ implies the following three inequalities. Since $n_{1} \alpha_{1}=n_{3} \alpha_{13}+n_{4} \alpha_{14}>n_{1} \alpha_{13}+n_{1} \alpha_{14}=n_{1}\left(\alpha_{13}+\alpha_{14}\right)$, we have $\alpha_{1}>\alpha_{13}+\alpha_{14}$. In the same manner, $\alpha_{4}<\alpha_{42}+\alpha_{43}$ and $\alpha_{3}<\alpha_{31}+\alpha_{32}$.

Remark 2.6. The extra condition $\alpha_{2} \leq \alpha_{21}+\alpha_{24}$ in Proposition 2.4 implies another condition on the generator $f_{5}$ in the following manner:

$$
\begin{aligned}
\alpha_{2}=\alpha_{32}+\alpha_{42} & \leq \alpha_{21}+\alpha_{24}, \\
\alpha_{32}+\alpha_{42}+\alpha_{14} & \leq \alpha_{21}+\alpha_{24}+\alpha_{14}=\alpha_{21}+\alpha_{4}, \\
\text { (since } \left.\alpha_{4}<\alpha_{42}+\alpha_{43}\right) \alpha_{32}+\alpha_{14} & <\alpha_{43}+\alpha_{21} .
\end{aligned}
$$

In order to prove Proposition 2.4, we need the following lemma. For the definitions of local orderings, normal form, ecart of a polynomial, standard basis and the description of the standard basis algorithm, see [8].

Lemma 2.7. The set $G=\left\{f_{1}, f_{2}, f_{3}, f_{4}, f_{5}\right\}$, where the polynomials $f_{i}$ are as in Proposition 2.4 with $\alpha_{2} \leq \alpha_{21}+\alpha_{24}$ is a standard basis with respect to the negative degree reverse lexicographical ordering with $x_{1}>x_{2}>x_{3}>x_{4}$.

Proof. We apply the standard basis algorithm to the set $G=\left\{f_{1}, f_{2}, f_{3}, f_{4}, f_{5}\right\}$. By using the notation in $[8$, we denote the leading monomial of a polynomial $f$ by $\operatorname{LM}(f)$, the s-polynomial of the polynomials $f$ and $g$ by spoly $(f, g)$ and the Mora's polynomial weak normal form of $f$ with respect to $G$ by $N F(f \mid G)$. We need to show that $N F\left(\operatorname{spoly}\left(f_{i}, f_{j}\right) \mid G\right)=0$ for all $i, j$ with $1 \leq i<j \leq 5$.

By using the inequalities in Remarks 2.5 and 2.6 with the extra condition $\alpha_{2} \leq$ $\alpha_{21}+\alpha_{24}$ and recalling that the ordering is the negative degree reverse lexicographical ordering, we have $\operatorname{LM}\left(f_{1}\right)=x_{3}^{\alpha_{13}} x_{4}^{\alpha_{14}}, \operatorname{LM}\left(f_{2}\right)=x_{2}^{\alpha_{2}}, \operatorname{LM}\left(f_{3}\right)=x_{3}^{\alpha_{3}}, \operatorname{LM}\left(f_{4}\right)=$ $x_{4}^{\alpha_{4}}$ and $\operatorname{LM}\left(f_{5}\right)=x_{2}^{\alpha_{32}} x_{4}^{\alpha_{14}}$. Since $\operatorname{lcm}\left(\operatorname{LM}\left(f_{1}\right), \operatorname{LM}\left(f_{2}\right)\right)=\operatorname{LM}\left(f_{1}\right) \cdot \operatorname{LM}\left(f_{2}\right)$, then $N F\left(\operatorname{spoly}\left(f_{1}, f_{2}\right) \mid\left\{f_{1}, f_{2}\right\}\right)=0$. This implies that $N F\left(\operatorname{spoly}\left(f_{1}, f_{2}\right) \mid G\right)=$ 0 . In the same manner, $N F\left(\operatorname{spoly}\left(f_{2}, f_{3}\right) \mid G\right)=0, N F\left(\operatorname{spoly}\left(f_{2}, f_{4}\right) \mid G\right)=0$, $N F\left(\operatorname{spoly}\left(f_{3}, f_{4}\right) \mid G\right)=0$ and $N F\left(\operatorname{spoly}\left(f_{3}, f_{5}\right) \mid G\right)=0$.

Next, we compute $\operatorname{spoly}\left(f_{1}, f_{3}\right)=x_{1}^{\alpha_{1}} x_{3}^{\alpha_{43}}-x_{1}^{\alpha_{31}} x_{2}^{\alpha_{32}} x_{4}^{\alpha_{14}}$. From Remark [2.6. $\alpha_{32}+\alpha_{14}<\alpha_{43}+\alpha_{21}$ and adding $\alpha_{31}$ to both sides of the inequality, we have $\alpha_{1}+\alpha_{43}>\alpha_{31}+\alpha_{32}+\alpha_{14}$, which implies $\operatorname{LM}\left(\operatorname{spoly}\left(f_{1}, f_{3}\right)\right)=x_{1}^{\alpha_{31}} x_{2}^{\alpha_{32}} x_{4}^{\alpha_{14}}$. Among 
the leading monomials of the elements of $G$, only $\operatorname{LM}\left(f_{5}\right)$ divides $\operatorname{LM}\left(\operatorname{spoly}\left(f_{1}, f_{3}\right)\right)$. (Also, ecart $\left(f_{5}\right)=\operatorname{ecart}\left(\operatorname{spoly}\left(f_{1}, f_{3}\right)\right)$.) The computation $\operatorname{spoly}\left(f_{5}, \operatorname{spoly}\left(f_{1}, f_{3}\right)\right)=$ 0 implies $N F\left(\operatorname{spoly}\left(f_{1}, f_{3}\right) \mid G\right)=0$.

Then, we compute $\operatorname{spoly}\left(f_{1}, f_{4}\right)=x_{1}^{\alpha_{1}} x_{4}^{\alpha_{24}}-x_{2}^{\alpha_{42}} x_{3}^{\alpha_{3}}$. The following inequalities

$$
\begin{aligned}
\alpha_{2} & \leq \alpha_{21}+\alpha_{24}, \\
\alpha_{2}+\alpha_{31} & \leq \alpha_{21}+\alpha_{31}+\alpha_{24}=\alpha_{1}+\alpha_{24}, \\
\left(\text { since } \alpha_{3}<\alpha_{31}+\alpha_{32}\right) \alpha_{2}+\alpha_{3}-\alpha_{32} & <\alpha_{1}+\alpha_{24}, \\
\alpha_{3}+\alpha_{42} & <\alpha_{1}+\alpha_{24}
\end{aligned}
$$

imply that $\operatorname{LM}\left(\operatorname{spoly}\left(f_{1}, f_{4}\right)\right)=x_{2}^{\alpha_{42}} x_{3}^{\alpha_{3}}$. Among the leading monomials of the elements of $G$, only $\operatorname{LM}\left(f_{3}\right)$ divides $\operatorname{LM}\left(\operatorname{spoly}\left(f_{1}, f_{4}\right)\right)$. (Also, we check that ecart $\left(f_{3}\right)$ $\leq$ ecart $\left(\operatorname{spoly}\left(f_{1}, f_{4}\right)\right)$.) We compute $\operatorname{spoly}\left(f_{3}, \operatorname{spoly}\left(f_{1}, f_{4}\right)\right)=x_{1}^{\alpha_{1}} x_{4}^{\alpha_{24}}-x_{1}^{\alpha_{31}} x_{2}^{\alpha_{2}}$. From (2.1), we have $\alpha_{2}+\alpha_{31} \leq \alpha_{1}+\alpha_{24}$, which gives $\operatorname{LM}\left(\operatorname{spoly}\left(f_{3}, \operatorname{spoly}\left(f_{1}, f_{4}\right)\right)\right)=$ $x_{1}^{\alpha_{31}} x_{2}^{\alpha_{2}}$. Once again, only $\operatorname{LM}\left(f_{2}\right)$ divides $\operatorname{LM}\left(\operatorname{spoly}\left(f_{3}, \operatorname{spoly}\left(f_{1}, f_{4}\right)\right)\right)$ among the leading monomials of the elements of the set $G$. (Notice also that ecart $\left(f_{2}\right)=$ $\operatorname{ecart}\left(\operatorname{spoly}\left(f_{3}, \operatorname{spoly}\left(f_{1}, f_{4}\right)\right)\right)$.) $\quad \operatorname{Since} \operatorname{spoly}\left(f_{2}, \operatorname{spoly}\left(f_{3}, \operatorname{spoly}\left(f_{1}, f_{4}\right)\right)\right)=0$, we have $N F\left(\operatorname{spoly}\left(f_{1}, f_{4}\right) \mid G\right)=0$.

Similarly, we compute $\operatorname{spoly}\left(f_{1}, f_{5}\right)=x_{1}^{\alpha_{1}} x_{2}^{\alpha_{32}}-x_{1}^{\alpha_{21}} x_{3}^{\alpha_{3}}$. Since $\alpha_{3}<\alpha_{31}+\alpha_{32}$, adding $\alpha_{21}$ to both sides of the inequality gives $\alpha_{3}+\alpha_{21}<\alpha_{32}+\alpha_{31}+\alpha_{21}=\alpha_{32}+\alpha_{1}$, which implies that $\operatorname{LM}\left(\operatorname{spoly}\left(f_{1}, f_{5}\right)\right)=x_{1}^{\alpha_{21}} x_{3}^{\alpha_{3}}$. Among the leading monomials of the elements of $G$, only $\operatorname{LM}\left(f_{3}\right)$ divides $\operatorname{LM}\left(\operatorname{spoly}\left(f_{1}, f_{5}\right)\right)$. (Also, ecart $\left(f_{3}\right)=$ ecart $\left(\operatorname{spoly}\left(f_{1}, f_{5}\right)\right)$.) Since $\operatorname{spoly}\left(f_{3}, \operatorname{spoly}\left(f_{1}, f_{5}\right)\right)=0, N F\left(\operatorname{spoly}\left(f_{1}, f_{5}\right) \mid G\right)=0$.

In the same manner, $\operatorname{spoly}\left(f_{2}, f_{5}\right)=x_{1}^{\alpha_{21}} x_{2}^{\alpha_{22}} x_{3}^{\alpha_{43}}-x_{1}^{\alpha_{21}} x_{4}^{\alpha_{4}}$. Since $\alpha_{4}<\alpha_{42}+$ $\alpha_{43}$ implies $\alpha_{4}+\alpha_{21}<\alpha_{42}+\alpha_{43}+\alpha_{21}$, then $\operatorname{LM}\left(\operatorname{spoly}\left(f_{2}, f_{5}\right)\right)=x_{1}^{\alpha_{21}} x_{4}^{\alpha_{4}}$. Among the leading monomials of the elements of $G$, only $\operatorname{LM}\left(f_{4}\right)$ divides $\operatorname{LM}\left(\operatorname{spoly}\left(f_{2}, f_{5}\right)\right)$. (Also, again ecart $\left(f_{4}\right)=\operatorname{ecart}\left(\operatorname{spoly}\left(f_{2}, f_{5}\right)\right)$.) $\operatorname{Since} \operatorname{spoly}\left(f_{4}, \operatorname{spoly}\left(f_{2}, f_{5}\right)\right)=0$, $N F\left(\operatorname{spoly}\left(f_{2}, f_{5}\right) \mid G\right)=0$.

Finally, spoly $\left(f_{4}, f_{5}\right)=x_{1}^{\alpha_{21}} x_{3}^{\alpha_{43}} x_{4}^{\alpha_{24}}-x_{2}^{\alpha_{2}} x_{3}^{\alpha_{43}}$ and since $\alpha_{2} \leq \alpha_{21}+\alpha_{24}$ implies $\alpha_{2}+\alpha_{43} \leq \alpha_{21}+\alpha_{24}+\alpha_{43}, \operatorname{LM}\left(\operatorname{spoly}\left(f_{4}, f_{5}\right)\right)=x_{2}^{\alpha_{2}} x_{3}^{\alpha_{43}}$. Among the leading monomials of the elements of $G$, only $\operatorname{LM}\left(f_{2}\right)$ divides $\operatorname{LM}\left(\operatorname{spoly}\left(f_{4}, f_{5}\right)\right)$. (Also, $\operatorname{ecart}\left(f_{2}\right)=\operatorname{ecart}\left(\operatorname{spoly}\left(f_{4}, f_{5}\right)\right)$.) Since $\operatorname{spoly}\left(f_{2}, \operatorname{spoly}\left(f_{4}, f_{5}\right)\right)=0$, again we have $N F\left(\operatorname{spoly}\left(f_{4}, f_{5}\right) \mid G\right)=0$.

Therefore, we conclude that the set $G$ is a standard basis with respect to the negative degree reverse lexicographical ordering with $x_{1}>x_{2}>x_{3}>x_{4}$.

We can now prove Proposition 2.4 .

Proof of Proposition 2.4. By Lemma 2.7 $G=\left\{f_{1}, f_{2}, f_{3}, f_{4}, f_{5}\right\}$, where the $f_{i}$ are as in Theorem 2.1. is a standard basis of $I(C)$ with respect to a local degree ordering (negative degree reverse lexicographic ordering with $x_{1}>x_{2}>x_{3}>x_{4}$ ). Then, from [8, Lemma 5.5.11], $I(C)_{*}$ is generated by the least homogeneous summands of the elements in the standard basis. Thus, $I(C)_{*}$ is generated by

$$
G_{*}=\left\{x_{3}^{\alpha_{13}} x_{4}^{\alpha_{14}}, x_{2}^{\alpha_{2}}, x_{3}^{\alpha_{3}}, x_{4}^{\alpha_{4}}, x_{2}^{\alpha_{32}} x_{4}^{\alpha_{14}}\right\}
$$

for $\alpha_{2}<\alpha_{21}+\alpha_{24}$ and by

$$
G_{*}=\left\{x_{3}^{\alpha_{13}} x_{4}^{\alpha_{14}}, x_{2}^{\alpha_{2}}-x_{1}^{\alpha_{21}} x_{4}^{\alpha_{24}}, x_{3}^{\alpha_{3}}, x_{4}^{\alpha_{4}}, x_{2}^{\alpha_{32}} x_{4}^{\alpha_{14}}\right\}
$$

for $\alpha_{2}=\alpha_{21}+\alpha_{24}$. 
Now, we can show that the Hilbert function of the Gorenstein local ring associated to the monomial curve $C$, with its defining ideal $I(C)$ as in Proposition 2.4 under the restriction given in the same proposition is non-decreasing.

Theorem 2.8. The Gorenstein monomial curve $C$ having parametrization as in (1.2) with $n_{1}<n_{2}<n_{3}<n_{4}$ and the defining ideal $I(C)$ as in Proposition 2.4 under the restriction $\alpha_{2} \leq \alpha_{21}+\alpha_{24}$ has Cohen-Macaulay tangent cone at the origin. Therefore, the Hilbert function of the associated Gorenstein local ring $k\left[\left[t^{n_{1}}, t^{n_{2}}, t^{n_{3}}, t^{n_{4}}\right]\right]$ is non-decreasing.

Proof. The Cohen-Macaulayness of the ring $k\left[x_{1}, x_{2}, x_{3}, x_{4}\right] / I(C)_{*}$ is obvious for the case $\alpha_{2}<\alpha_{21}+\alpha_{24}$, since in this case, the generators of $I(C)_{*}$ are all monomials not divisible by $x_{1}$ from Proposition 2.4. For the case $\alpha_{2}=\alpha_{21}+\alpha_{24}$, we can apply the theorem in [1, Theorem 2.1] to the generators of $I(C)_{*}$ given by

$$
G_{*}=\left\{x_{3}^{\alpha_{13}} x_{4}^{\alpha_{14}}, x_{2}^{\alpha_{2}}-x_{1}^{\alpha_{21}} x_{4}^{\alpha_{24}}, x_{3}^{\alpha_{3}}, x_{4}^{\alpha_{4}}, x_{2}^{\alpha_{32}} x_{4}^{\alpha_{14}}\right\}
$$

in Proposition 2.4. This is a Gröbner basis with respect to the reverse lexicographic order with $x_{1}>x_{4}>x_{2}>x_{3}$. Since $x_{1}$ does not divide the leading monomial of any element in $G_{*}$, we conclude from [1, Theorem 2.1] that $x_{1}$ is not a zero-divisor and thus, the ring $k\left[x_{1}, x_{2}, x_{3}, x_{4}\right] / I(C)_{*}$ is Cohen-Macaulay. Therefore, since $\operatorname{gr}_{m}\left(k\left[\left[t^{n_{1}}, t^{n_{2}}, t^{n_{3}}, t^{n_{4}}\right]\right]\right) \cong k\left[x_{1}, x_{2}, x_{3}, x_{4}\right] / I(C)_{*}$ is Cohen-Macaulay, the Hilbert function of the Gorenstein local ring $k\left[\left[t^{n_{1}}, t^{n_{2}}, t^{n_{3}}, t^{n_{4}}\right]\right]$ is non-decreasing.

Remark 2.9. Since we have the condition $n_{1}<n_{2}<n_{3}<n_{4}$ in Proposition 2.4 and Theorem 2.8, we have to consider the other permutations of the defining ideal to generalize this result. The same method can be applied to the following cases in Remark 2.3 to obtain similar results. By using the same algorithm in Lemma 2.7. we have a standard basis with respect to the negative degree reverse lexicographical ordering with $x_{1}>x_{2}>x_{3}>x_{4}$ :

- In Case 1(b) with the restriction $\alpha_{2} \leq \alpha_{21}+\alpha_{23}, \alpha_{3} \leq \alpha_{32}+\alpha_{34}$;

- In Case 2(b) with the restriction $\alpha_{2} \leq \alpha_{21}+\alpha_{24}, \alpha_{3} \leq \alpha_{32}+\alpha_{34}$;

- In Case 3(a) with the restriction $\alpha_{2} \leq \alpha_{21}+\alpha_{23}, \alpha_{3} \leq \alpha_{31}+\alpha_{34}$.

Thus, in all these cases, $I(C)_{*}$ is generated by a set of five elements, which are the homogeneous summands of least degree of the five generators of $I(C)$. By using the same method in Theorem 2.8, we show that the Gorenstein monomial curves having the defining ideals as in these cases with the given conditions have CohenMacaulay tangent cones. We do not have a similar result in Cases 2 (a) and 3 (b), since $f_{2}=x_{2}^{\alpha_{2}}-x_{3}^{\alpha_{23}} x_{4}^{\alpha_{24}}$ is an element of the generator set in both cases, and thus, $n_{1}<n_{2}<n_{3}<n_{4}$ implies $\alpha_{2}>\alpha_{23}+\alpha_{24}$.

As a result, we can now give the general theorem:

Theorem 2.10. Let $C$ be a Gorenstein monomial curve with embedding dimension four having parametrization

$$
x_{1}=t^{n_{1}}, x_{2}=t^{n_{2}}, x_{3}=t^{n_{3}}, x_{4}=t^{n_{4}}
$$

where $n_{1}<n_{2}<n_{3}<n_{4}$ and let the generators of the defining ideal $I(C)$ (given by one of the cases in Remark 2.3) be denoted as $f_{1}=x_{1}^{\alpha_{1}}-m_{1}, f_{2}=x_{2}^{\alpha_{2}}-m_{2}, f_{3}=$ $x_{3}^{\alpha_{3}}-m_{3}, f_{4}=x_{4}^{\alpha_{4}}-m_{4}$ and $f_{5}$, where $m_{1}, m_{2}, m_{3}$ and $m_{4}$ are monomials. If $\alpha_{2} \leq$ total degree of $m_{2}$ and $\alpha_{3} \leq$ total degree of $m_{3}$, then the Hilbert function of the local Gorenstein ring $k\left[\left[t^{n_{1}}, t^{n_{2}}, t^{n_{3}}, t^{n_{4}}\right]\right]$ is non-decreasing. 
Proof. The proof is a direct consequence of Theorem 2.8 and Remark 2.9

2.1. A family of examples. We can now construct a family of Gorenstein monomial curves satisfying the conditions of Theorem 2.8, and thus having associated Gorenstein local rings with non-decreasing Hilbert function. The construction is done by using Bresinsky's characterization of the $n_{i}$ 's in terms of the $\alpha_{i}$ 's and $\alpha_{i j}$ 's given in Remark 2.2 and considering the extra conditions in Theorem 2.8.

Let $C_{m}$ be a monomial curve having parametrization

$$
x_{1}=t^{n_{1}(m)}, x_{2}=t^{n_{2}(m)}, x_{3}=t^{n_{3}(m)}, x_{4}=t^{n_{4}(m)}
$$

with $n_{1}(m)=m^{3}+m^{2}-m, n_{2}(m)=m^{3}+2 m^{2}+m-1, n_{3}(m)=m^{3}+3 m^{2}+$ $2 m-2, n_{4}(m)=m^{3}+4 m^{2}+3 m-2$ where $m \geq 2$ is an integer.

It is trivial to check that $n_{1}(m)<n_{2}(m)<n_{3}(m)<n_{4}(m)$ for $m \geq 2$. Also, $\operatorname{gcd}\left(n_{1}(m), n_{2}(m), n_{3}(m), n_{4}(m)\right)=1$ for $m \geq 2$, since $n_{4}(m)-2 n_{3}(m)+$ $n_{2}(m)=1$. By using Bresinsky's characterization of the $n_{i}$ 's in terms of the $\alpha_{i}$ 's and $\alpha_{i j}$ 's given in Remark 2.2, we conclude that the numerical semigroup $S_{m}=\left\langle n_{1}(m), n_{2}(m), n_{3}(m), n_{4}(m)\right\rangle$ is symmetric, and moreover $I\left(C_{m}\right)$ is generated by the set

$$
\begin{aligned}
G_{m}=\left\{f_{1}=\right. & x_{1}^{m+3}-x_{3} x_{4}^{m-1}, f_{2}=x_{2}^{m+2}-x_{1}^{m+2} x_{4}, f_{3}=x_{3}^{m}-x_{1} x_{2}^{m}, \\
f_{4}= & \left.x_{4}^{m}-x_{2}^{2} x_{3}^{m-1}, f_{5}=x_{3}^{m-1} x_{1}^{m+2}-x_{2}^{m} x_{4}^{m-1}\right\} .
\end{aligned}
$$

It is obvious that $I\left(C_{m}\right)$ satisfies the conditions of Theorem 2.8 for all integers $m \geq 2$, and thus, the defining ideal of the tangent cone $I\left(C_{m}\right)_{*}$ is generated by the set

$$
G_{m_{*}}=\left\{x_{3} x_{4}^{m-1}, x_{2}^{m+2}, x_{3}^{m}, x_{4}^{m}, x_{2}^{m} x_{4}^{m-1}\right\} .
$$

Since $\operatorname{gr}_{m}\left(k\left[\left[t^{n_{1}(m)}, t^{n_{2}(m)}, t^{n_{3}(m)}, t^{n_{4}(m)}\right]\right]\right) \cong k\left[x_{1}, x_{2}, x_{3}, x_{4}\right] / I\left(C_{m}\right)_{*}$ is CohenMacaulay, the Gorenstein local ring $k\left[\left[t^{n_{1}(m)}, t^{n_{2}(m)}, t^{n_{3}(m)}, t^{n_{4}(m)}\right]\right]$ has a nondecreasing Hilbert function for all $m \geq 2$.

By using the characterization in Remark 2.2, many families can be constructed in the same manner, and thus, we can obtain large families of Gorenstein local rings with embedding dimension four having non-decreasing Hilbert functions.

\section{Complete intersection CASe}

In this section, we give a general result for the complete intersection case, which gives a constructive method to obtain large families of complete intersection local rings with arbitrary embedding dimension having non-decreasing Hilbert functions. To do this, we first generalize the family of numerical semigroups given in [3].

Definition 3.1. Let $H=\left\langle n_{1}, \ldots, n_{d}\right\rangle$ be a numerical semigroup minimally generated by $n_{1}, \ldots, n_{d}$. A numerical semigroup $H^{\prime}$ is an extension of $H$ if there is an element $n_{d+1}=a_{1} n_{1}+\ldots+a_{d} n_{d} \in H$ other than $n_{1}, \ldots, n_{d}$ and an integer $p$ with $\operatorname{gcd}\left(p, n_{d+1}\right)=1$ such that

$$
H^{\prime}=\left\langle p n_{1}, \ldots, p n_{d}, n_{d+1}\right\rangle .
$$

$H^{\prime}$ is a nice extension of $H$ if in addition $p \leq a_{1}+\ldots+a_{d}$.

Remark 3.2. The conditions $\operatorname{gcd}\left(p, n_{d+1}\right)=1$ and $n_{d+1}$ is different than the generators of $H$ imply that $H^{\prime}$ is minimally generated by $p n_{1}, \ldots, p n_{d}, n_{d+1}$. 
Lemma 3.3. Let $H^{\prime}$ be an extension of the numerical semigroup $H$ with the associated monomial curves $C^{\prime}$ and $C$. If $I(C)$ is generated by the set $G$, then $I\left(C^{\prime}\right)$ is generated by $G \cup\left\{x_{d+1}^{p}-x_{1}^{a_{1}} \cdots x_{d}^{a_{d}}\right\}$. In particular, if $C$ is a complete intersection, so is $C^{\prime}$.

Proof. The proof is a direct consequence of the two lemmas of Morales in [15, Lemmas 1.3 and 1.5].

Definition 3.4. A numerical semigroup $H$ is obtained by a sequence of (nice) extensions if there are numerical semigroups $H_{0}, \ldots, H_{d}$ such that $H_{0}=\mathbb{N}, H_{d}=H$ and $H_{i+1}$ is a (nice) extension of $H_{i}$ for $i=0, \ldots, d-1$.

Obviously, every monomial curve associated with a numerical semigroup obtained by a sequence of extensions is a complete intersection from Lemma 3.3. In fact, we can give the generators explicitly as in the following lemma.

Lemma 3.5. Let $H=H_{d}$ with $d \geq 2$ be a numerical semigroup obtained by a sequence of extensions and $C$ be the associated monomial curve. Then $I(C)$ is generated minimally by a set of the form

$$
\left\{x_{2}^{p_{2}}-m_{2}, \ldots, x_{d}^{p_{d}}-m_{d}\right\}
$$

where $m_{i}$ is a monomial in $x_{1}, \ldots, x_{i-1}$ for $i=2, \ldots, d$. Moreover, if $H$ is obtained by a sequence of nice extensions, then $p_{i} \leq$ total degree of $m_{i}$ for $i=2, \ldots, d$.

Proof. The proof can be done easily by induction on $d$ and using Lemma 3.3. The second part follows from the definition of a nice extension.

By using the definitions given, we recall the results concerning the structure of complete intersection numerical semigroups. Every complete intersection numerical semigroup with 3 generators is obtained by a sequence of extensions [11, but for 4 generators, this is no longer true [3], and the complete intersection monomial curves given in [3, Theorem 2] are exactly the ones associated to numerical semigroups with 4 generators obtained by a sequence of extensions. Also, the general structure of complete intersection numerical semigroups was given by Delorme 4 .

We can now give our main result.

Theorem 3.6. Let $H=\left\langle n_{1}, \ldots, n_{d}\right\rangle$ be a numerical semigroup obtained by a sequence of nice extensions, and let $C$ be the associated monomial curve. Then the tangent cone of $C$ at the origin is Cohen-Macaulay, and hence the Hilbert function of the Gorenstein local ring $k\left[\left[t^{n_{1}}, \ldots, t^{n_{d}}\right]\right]$ is non-decreasing.

Proof. The proof is very similar to the proof of Theorem 2.8, If $d=1$, the result is obvious. If $d \geq 2$, then from Lemma 3.5, $I(C)$ is generated minimally by the set $G=\left\{x_{2}^{p_{2}}-m_{2}, \ldots, x_{d}^{p_{d}}-m_{d}\right\}$, where $m_{i}$ is a monomial in $x_{1}, \ldots, x_{i-1}$ and $p_{i} \leq$ total degree of $m_{i}$ for $i=2, \ldots, d$. The conditions on the integers $p_{i}$ imply that $n_{1}$ is the smallest integer among the generators of $H$, so we must show that $x_{1}$ is not a zero-divisor in $k\left[x_{1}, \ldots, x_{d}\right] / I(C)_{*}$. The set $G$ is a standard basis with respect to the negative degree reverse lexicographical ordering with $x_{d}>\ldots>x_{1}$, since $\operatorname{LM}\left(x_{2}^{p_{2}}-m_{2}\right)=x_{2}^{p_{2}}, \ldots, \operatorname{LM}\left(x_{d}^{p_{d}}-m_{d}\right)=x_{d}^{p_{d}}$ and no two of them have a common divisor of total degree greater than or equal to 1 . Thus, $I(C)_{*}$ is generated by the set $G_{*}$ consisting of the least homogeneous summands of the elements in $G$. The set $G_{*}$ is a Gröbner basis with respect to the reverse lexicographic order with $x_{1}>\ldots>x_{d}$, and since $x_{1}$ does not divide the leading monomial of any element in 
$G_{*}$, we conclude from [1, Theorem 2.1] that $x_{1}$ is not a zero-divisor, which shows that $k\left[x_{1}, \ldots, x_{d}\right] / I(C)_{*}$ is Cohen-Macaulay. Therefore, the Hilbert function of the Gorenstein local ring $k\left[\left[t^{n_{1}}, \ldots, t^{n_{d}}\right]\right]$ is non-decreasing.

By using Theorem [3.6 and constructing families of numerical semigroups obtained by a sequence of nice extensions, we can present large families of complete intersection local rings with arbitrary embedding dimension having non-decreasing Hilbert functions.

\section{ACKNOWLEDGMENTS}

We would like to thank Juan Elias for bringing this problem to our attention. We also would like to thank the referee for very helpful suggestions.

\section{REFERENCES}

1. F. Arslan, Cohen-Macaulayness of tangent cones, Proc. Amer. Math. Soc. 128 (2000), 22432251. MR 1653409 (2000k:13021)

2. D. Bayer and M. Stillman, Macaulay, A system for computation in algebraic geometry and commutative algebra, 1992.

3. H. Bresinsky, Symmetric semigroups of integers generated by four elements, Manuscripta Math. 17, (1975), 205-219. MR0414559 (54:2660)

4. C. Delorme, Sous-monoïdes d'intersection complète de $N$, Ann. Sci. École Norm. Sup. (4) 9 No.1 (1976), 145-154. MR0407038 (53:10821)

5. J. Elias, The Conjecture of Sally on the Hilbert Function for Curve Singularities, Journal of Algebra 160 No.1 (1993), 42-49. MR.1237076 (94j:13018)

6. J. Elias, Private communication.

7. J. Eakin, A. Sathaye, Prestable ideals, Journal of Algebra 41 (1976), 439-454. MR0419428 $(54: 7449)$

8. G.-M. Greuel, G. Pfister, A Singular Introduction to Commutative Algebra, Springer-Verlag, 2002. MR 1930604 (2003k:13001)

9. G.-M. Greuel, G. Pfister, and H. Schönemann. Singular 2.0. A Computer Algebra System for Polynomial Computations. Centre for Computer Algebra, University of Kaiserslautern (2001). http://www.singular.uni-kl.de.

10. S.K. Gupta, L.G. Roberts, Cartesian squares and ordinary singularities of curves, Comm. in Algebra 11 No.2 (1983), 127-182. MR0688046 (84h:13036)

11. J. Herzog, Generators and relations of abelian semigroups and semigroup rings, Manuscripta Math. 3 (1970), 175-193. MR0269762 (42:4657)

12. J. Herzog, R. Waldi, A note on the Hilbert function of a one-dimensional Cohen-Macaulay ring, Manuscripta Math. 16, (1975), 251-260. MR0384785 (52:5658)

13. E. Kunz, The value-semigroup of a one-dimensional Gorenstein ring, Proc. Amer. Math. Soc. 25 (1970), 748-751. MR0265353 (42:263)

14. E. Matlis, One-dimensional Cohen-Macaulay Rings, Lecture Notes in Mathematics 327, Springer-Verlag, 1977. MR0357391 (50:9859)

15. M. Morales, Syzygies of a monomial curve and a linear Diophantine problem of Frobenius, Max-Planck-Institut für Mathematik, preprint, 1987.

16. O. Orecchia, One-dimensional local rings with reduced associated graded ring and their Hilbert functions, Manuscripta Math. 32 (1980), 391-405. MR0595429 (83c:13011)

17. L.G. Roberts, Ordinary singularities with decreasing Hilbert function, Canad. J. Math. 34 (1982), 169-180. MR0650856 (83f:13007)

18. J. Sally, Number of generators of ideals in local rings, Lecture Notes in Pure and Appl. Math. 35, Marcel Dekker, 1978. MR0485852 (58:5654)

19. G. Valla, Problems and results on Hilbert functions of graded algebras, Six Lectures on Commutative Algebra, Progr. Math. vol. 66, Birkhäuser, Basel, 1998, pp. 293-344. MR 1648668 (99k:13022) 
Department of Mathematics, Middle East Technical University, Ankara, 06531 TURKEY

E-mail address: sarslan@metu.edu.tr

Department of Mathematics, Middle East Technical University, Ankara, 06531 TURKEY

E-mail address: pinarm@metu.edu.tr

Current address: Department of Mathematics, Balıkesir University, Balıkesir, 10145 Turkey

E-mail address: pinarm@balikesir.edu.tr 